\title{
Electrospun Silybin Enriched Scaffolds of Polyethylene Oxide as Wound Dressings: Enhanced Wound Closure, Reepithelization in Rat Excisional Wound Model
}

\author{
Prajakta Deepak Kapadnis, Shilpa Nilesh Shrotriya* \\ Department of Pharmaceutics, Sinhgad College of Pharmacy, S. No. 44/1, Vadgaon (BK), Off Sinhgad road, Pune-411041, \\ Maharashtra, INDIA.
}

\begin{abstract}
Aim/ Background: Wounds are considered as a common health problem due to their considerable burden on social life. Polymeric nanofibers have attracted attention in wound dressings due to their large surface area, smaller size and biocompatibility. The objective of this research is to develop silybin loaded polymeric scaffolds and further to check its efficacy in wound healing. Materials and Methods: Silybin loaded polymeric scaffolds was developed using polyethylene oxide as a polymer and glutaraldehyde as a crosslinker by electrospinning technique. It was characterized for scanning electron microscopy, differential scanning calorimetry, X-ray diffraction, swelling behavior, water uptake capacity and drug release kinetics. Further, in vivo studies were carried out on rats by excisional wound model. Histopathological studies were performed to investigate the healing process. Results: From the results of scanning electron microscopy, $3 \%$ Polyethylene oxide in dichloromethane: dimethyl formamide (8:2) mixture was selected for further characterization. Swelling index for prepared electrospun scaffolds was found to be $62.40 \%$, whereas water uptake capacity was found to be $38.42 \%$. Release kinetics suggested sustained release of silybin. The histopathological study indicated that silybin loaded scaffolds improved the results for granulation tissue score, wound maturity score, period of epithelisation and collagen distribution. Conclusion: It can be concluded that silybin enriched nanofibrous scaffold of polyethylene oxide is beneficial in topical applications to form the basis for new skin regenerating and wound healing.
\end{abstract}

Key words: Nanofiber, Electrospinning, Scaffold, Silybin, Wound healing.

\section{INTRODUCTION}

Ideally a wound dressing should facilitate and accelerate wound healing at reasonable cost and dressing should be removed easily without causing any pain to the patient. ${ }^{1,2}$ Wound dressings restore the ideal environment for skin regeneration and protect wounds from environmental hazards and bacterial invasion. ${ }^{3}$ Local delivery of drugs is preferred to systemic administration as these systems have several advantages like improving the effectiveness of therapy, selective targeting to the site, decreasing the side effects on a tissue and reducing the frequency of dressing replacements.
Furthermore, a wound dressing needs to have ability to maintain moist environment around the wound bed. ${ }^{2,4}$ Dressings are used as vehicles for delivering therapeutic agents to wound sites. A wide range of wound dressings are applied for variety of wounds and to target different stages of wound healing., ${ }^{4,5}$ Formulations like creams, gels, dry traditional dressings like cotton wool and gauze, hydrogels and hydrocolloids are commonly used as wound dressings. Recently, nanofibrous layered dressings from suitable polymers are widely used for wound dressing applications. ${ }^{6}$
Submission Date: 03-07-2018; Revision Date: 31-10-2018; Accepted Date: 29-12-2018

DOI: 10.5530/ijper.53.2.38 Correspondence: Mrs. Shilpa Nilesh Shrotriya, Department of Pharmaceutics, Sinhgad College of Pharmacy, S. No. 44/1, Vadgaon (BK), Off Sinhgad road,

Pune-411041. Maharashtra, INDIA.

Phone: +91-9850449044 E-mail: snshrotriya2014@ gmail.com

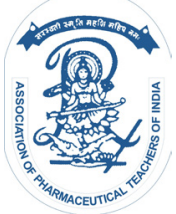

www.ijper.org 
Presently, polymeric nanofibers have attracted attention due to their unique features like large surface area, high porosity, smaller pore size and ability to incorporate large variety of biomolecules. ${ }^{2,7}$ Nanofibers can be used for several applications such as wound dressings, tissue engineering and drug delivery. ${ }^{8}$ The very fine diameter and highly porous structure of nanofiber matrix help drug particles diffuse out of the scaffold more efficiently. Electrospinning is simple, versatile and inexpensive technique for fabrication of polymeric nanofibers. ${ }^{9}$ Use of electrospun nanofiber scaffolds for wound dressings also has some benefits, such as absorbability of wound exudate and acceleration of wound healing process. ${ }^{7}$

Various natural and synthetic polymers like collagen, elastin, cellulose, cellulose acetate, chitosan, gelatin, polyvinyl alcohol, silk fibroin, polyethylene oxide are used in wound healing formulation. ${ }^{8,10}$ Polyethylene oxide (PEO) is a non-ionic homopolymer of ethylene oxide, which has high swelling capacity and can be effectively used in sustained-release matrix formulations. $\mathrm{PEO}$ is a biocompatible polymer which is commonly used in wound care applications. ${ }^{10}$ Crosslinking is necessary to control drug release from the polymeric structure as well as to impart mechanical properties to the scaffolds.

It has been proved that ROS-induced wounds may be cured through supplementation by antioxidants, such as phenolic compounds. In past years, protective activities of various herbal phenolic compounds namely phenolic acids and flavonoids have been investigated. Silymarin is the extract from seeds of milk thistle (Silybum marianum) which has been widely used in the treatment of liver disorders. ${ }^{11}$ Main active moiety found in extract is Silybin (SIL) which is chemically a flavonolignan compound. ${ }^{12}$ Topical application of SIL is shown to protect chemical and physical damage resulting from burns in mouse and humans. ${ }^{13}$ SIL possess antioxidant, anticancer and anti-inflammatory activity. It is also useful in irritant contact dermatitis. ${ }^{14}$ SIL induces number of endogenous antioxidants, including superoxide dismutase (SOD), glutathione peroxidase (GPx), glutathione reductase and glucose 6-phosphate dehydrogenase. It also inhibits COX-2 which inhibits the inflammatory damage to the wound site. ${ }^{13}$ Due to its strong antioxidant and anti-inflammatory activity, SIL can be effectively useful for cutaneous wound healing.

In present research, electrospinning was applied to fabricate silybin engrossed PEO scaffolds with high swelling capacity and controlled release of SIL for better wound care.

\section{MATERIALS AND METHODS}

\section{Materials}

Polyethylene oxide (PEO) (MW = $200000 \mathrm{Da})$ was obtained as a gift sample from Wockhardt (Aurangabad). Silybin (SIL) was purchased from Prolab, New Delhi. All the other chemicals were of analytical grade and used as obtained.

\section{Animals}

The study protocol was approved (SCOP/IAEC/201516/218) by the Institutional Animal Ethics Committee (IAEC) of Sinhgad College of Pharmacy, Pune, constituted under Committee for Purpose of Control and Supervision of Experiments on Animals (CPCSEA). Adult Wistar albino rats of either sex weighing $250 \pm 50 \mathrm{~g}$ were housed in plastic cages in the temperature controlled room $\left(22 \pm 2^{\circ} \mathrm{C}\right)$ with free access to food and water and were acclimated at least one week before experiments.

\section{HPLC method development for Silybin}

The quantitative determination of SIL was performed by High Performance Liquid Chromatography (HPLC) (Shimadzu, LC 2010) at $288 \mathrm{~nm}$. Briefly, samples were chromatographed on a $4.6 \mathrm{~mm} \times 250 \mathrm{~mm}$ reversed phase stainless steel column packed with $5 \mathrm{~mm}$ particles $\left(\right.$ Hypersil $\mathrm{C}_{18}$ ) and eluted with a mobile phase consisting of acetonitrile water and methanol in the ratio of $70: 25: 5 \mathrm{v} / \mathrm{v}$ at a flow rate of $1 \mathrm{ml} / \mathrm{min}$.

\section{Formulation of polymeric electrospun scaffolds}

The scaffolds were prepared by electrospinning method. ${ }^{15}$ The plain electrospun scaffolds (NF2-P, NF3-P and NF4-P) were prepared by dissolving 2\%, 3\% and 4\% PEO in dichloromethane: dimethyl formamide (DCM: DMF) mixture (8:2) at room temperature by electrospinning (ESPIN NANO, PECO-Chennai). Electrospun SIL engrossed scaffolds (SIL-NF2, SILNF3 and SIL-NF4) were prepared by using $2 \%, 3 \%$ and 4\% PEO in DCM: DMF mixture (8:2) with addition of SIL $(0.1 \% \mathrm{w} / \mathrm{w})$ to the polymer solutions. Composition of batches is given in Table 1. For electrospinning, the solutions were transferred to a $10 \mathrm{ml}$ syringe pump with a blunt end needle (gauge 22) attached to it. The resulting fibers were collected on a grounded aluminium collector plate. The solution flow rate was set to $1 \mathrm{ml} / \mathrm{h}$, the applied voltage was adjusted to $20 \mathrm{kV}$ and the distance between the needle tip and collector plate was fixed to $10 \mathrm{~cm}$. Prepared scaffolds were then crosslinked by treatment with glutaraldehyde vapours, saturated with $2 \%$ glutaraldehyde aqueous solution at room 


\begin{tabular}{|c|c|c|c|}
\hline \multicolumn{3}{|c|}{ Table 1: Formulation of polymeric scaffolds. } \\
\hline Batch code & $\begin{array}{c}\text { SIL } \\
(\mathbf{m g})\end{array}$ & PEO (\%) & $\begin{array}{c}\text { DCM: DMF } \\
\text { solvent } \\
\text { mixture }\end{array}$ \\
\hline Plain-NF2 scaffold & - & 2 & $8: 2$ \\
\hline Plain-NF3 scaffold & - & 3 & $8: 2$ \\
\hline Plain-NF4 scaffold & - & 4 & $8: 2$ \\
\hline SIL-NF2 scaffold & 25 & 2 & $8: 2$ \\
\hline SIL-NF3 scaffold & 25 & 3 & $8: 2$ \\
\hline SIL-NF4 scaffold & 25 & 4 & $8: 2$ \\
\hline
\end{tabular}

temperature for $48 \mathrm{~h}$ followed by treatment with $0.1 \mathrm{M}$ glycine aqueous solution. ${ }^{16}$

\section{Evaluation of prepared scaffolds \\ Scanning Electron Microscopy (SEM)}

Morphology of prepared porous scaffolds was observed under scanning electron microscope (SEM: Bruker $\mathrm{X}$-flash $6130, \mathrm{USA}$ at $25 \pm 2^{\circ} \mathrm{C}$ with accelerating voltage of $10 \mathrm{kV})$. Initially, all the samples were mounted on the specimen holder with electroconductive tape; sputter coated under vacuum with gold in a coating unit. The precoated samples were then analysed under microscope.

\section{Differential scanning calorimetry (DSC) studies}

The possibility of any interaction between drug and excipients was accessed by carrying out thermal analysis of formulation using DSC. The DSC thermogram of SIL, PEO, physical mixture and the optimized SIL-NF3 batch were recorded by using a differential scanning calorimeter (DSC: Perkin Elmer 4000, USA). DSC scans were recorded at heating rate of $10^{\circ} \mathrm{C} / \mathrm{min}$ in a temperature range of 25 to $300^{\circ} \mathrm{C}$ and $40 \mathrm{ml} / \mathrm{min}$ of nitrogen flow.

\section{X-ray diffraction studies (XRD)}

XRD of SIL, PEO, physical mixture and the optimized SIL-NF3 batch was performed on powder X-ray diffractometer (Bruker D8 Advance, Germany) with copper as an anode material and dermic $x$-ray tube as the source operated at a voltage of $30 \mathrm{kV}$ and a current of $30 \mathrm{~mA}$. The samples were analysed using lynux eye detector and filtered using Ni filter with $2 \theta$ angle ranging from 5 to $60^{\circ}$.

\section{Swelling behaviour and water uptake capacity}

To study swelling behaviour of scaffolds, $2 \times 2 \mathrm{~cm}$ scaffolds were weighed $\left(\mathrm{W}_{\mathrm{d}}\right)$ and were immersed in water for $24 \mathrm{~h}$. Swollen scaffolds were centrifuged at $500 \mathrm{rpm}$ in the presence of filter to remove excess water from the scaffolds and the wet weight $\left(\mathrm{W}_{\mathrm{s}}\right)$ was determined.
The degree of swelling (Q) and water uptake (\%) were calculated with equations (1) and (2) respectively. ${ }^{17}$

Degree of swelling $(\mathrm{Q})=\left(\mathrm{W}_{\mathrm{s}}-\mathrm{W}_{\mathrm{d}}\right) \div \mathrm{W}_{\mathrm{d}} \times 100$

Water uptake $(\%)=\mathrm{W}_{\mathrm{s}}-\mathrm{W}_{\mathrm{d}} \div \mathrm{W}_{\mathrm{s}} \times 100$

where,

$W_{d}$ - Weight of dry scaffold

$\mathrm{W}_{\mathrm{s}}$ - Weight of wet scaffold

\section{Drug release kinetics studies}

Silybin loaded scaffolds of dimensions $2 \times 2 \mathrm{~cm}$ were used for this study. The films were submerged in glass vessels containing $10 \mathrm{ml}$ of phosphate buffer saline, $\mathrm{pH}$ 7.4 (PBS) and incubated at $37^{\circ} \mathrm{C} \pm 2^{\circ} \mathrm{C}$ with stirring continuously at $150 \mathrm{rpm}$. After particular time intervals, $3 \mathrm{ml}$ of dissolution medium was collected and measured by high pressure liquid chromatography (HPLC). After collection of medium, $3 \mathrm{ml}$ of fresh PBS was added to the vessel to maintain the constant volume. The release was observed for $178 \mathrm{~h}$ after scaffolds immersion. ${ }^{18}$ To understand release kinetics, data was analyzed using PCP Disso software and fitted into four mathematical models like zero order, first order, Hixon-crowell and Korsmeyer-Peppas model.

\section{In-vivo animal Study: Excision wound model}

In-vivo excision wound model was employed to assess the wound closure in Wistar albino rats. Rats were anesthetized by ketamine hydrochloride $(60 \mathrm{mg} / \mathrm{kg}$ body weight) and the dorsal hair was shaved and a circle measuring $2 \times 2 \mathrm{~cm}$ was outlined in each animal with marker. The circular pieces of full thickness were removed by scalpel. Animals were divided into 4 groups (12 rats per group), wounds were topically treated with NS, Marketed formulation (containing povidone iodine), SIL loaded nanofibrous scaffolds (SIL-NF3), plain nanofibrous scaffolds (NF-P), respectively and the wounds were wrapped with surgical bandage to keep the scaffolds in place. For re-epithelization study, treatment was continued on another set of rats $(n=6)$ until epithelization was complete and endpoint of epithelization was observed. Period of epithelization was the number of days taken to achieve epithelization.

\section{Wound closure (\%)}

Wound closure $(\%)$ was considered as a criterion for investigating wound healing activity. ${ }^{19}$ The areas of wounds were measured by tracing the wounds on trace paper at the intervals of $3^{\text {th }}, 7^{\text {th }}$ and $14^{\text {th }}$ day of injury. The rate of wound closure was calculated by using following equation,

Wound closure $(\%)=\left(A_{0}-A_{t}\right) / A_{0} \times 100$ 
where,

$A_{0}$ - initial wound area

$A_{t}$ - wound area after a time interval t.

Wound healing process was photographed to observe wound closure.

\section{Histopathological evaluation}

On $15^{\text {th }}$ day, animals were sacrificed. The wounded skin surrounded by $2 \mathrm{~cm}$ of healthy was removed immediately from individual rats for histopathology. Samples were fixed in 10\% buffered formalin, dehydrated by alcohol and cleared in xylene. The tissue sections were embedded in paraffin and cut into $5 \mathrm{~mm}$ thick sections and mounted on glass microscope slides. The samples were analyzed by hematoxylin and eosin ( $\mathrm{H}$ and $\mathrm{E}$ ) staining and Masson's trichrome staining (MTS) and various wound parameters were evaluated.

\section{Reepithelization}

Sections were stained with hematoxylin and eosin ( $\mathrm{H}$ and $\mathrm{E})$ and epithelial layer was observed to evaluate histopathological alterations.

\section{Granular tissue score}

The granular tissue score was evaluated from histopathological results of wound after 14 days. The $\mathrm{H}$ and $\mathrm{E}$ stained sections of wound area were employed to observe granular tissue score by two pathologists in a blinded manner. The granulation tissue score was evaluated by using score system. (Score 1 - No granular tissue, Score 2 - Minimal granular tissue, Score 3 Moderate granular tissue, Score 4 - Extensive granular tissue score, Score 5 - Very extensive tissue)

\section{Wound maturity score}

Wound maturity score was evaluated on the basis of attentiveness of inflammatory cells on wound. The $\mathrm{H}$ and $\mathrm{E}$ stained sections of wound area were employed to observe inflammatory cells by two pathologists in a blinded manner. The wound maturity score was evaluated by using score system (Score 1 - Very extensive inflammatory cells, Score 2 - Extensive inflammatory cells, Score 3 - Moderate inflammatory cells, Score 4 - Minimal inflammatory cells, Score 5 - No inflammatory cells)

\section{Collagen distribution}

Wounded tissue sections were stained with Masson's trichrome stain (MTS) and collagen distribution was observed in histopathology.

\section{Statistical analysis}

Statistical analysis was performed using Graphpad Prism Software version 5.03. The results were statistically accessed by One Way and Two Way analysis of variance (ANOVA). Following a significant $P$ value, post hoc analysis (Tukey's test and Bonferroni t-test) were performed for multiple comparisons. The results are displayed as a mean value with a standard error of mean (mean \pm SEM). A level for $P<0.05$ and $P<0.01$ respectively were considered to be significant.

\section{RESULTS AND DISCUSSION}

\section{Formulation of scaffolds}

Polymeric scaffolds were successfully prepared by electrospinning process. Crosslinking of scaffolds with glutaraldehyde was done to increase the tensile strength of scaffolds. The as-spun PEO nanofibric scaffolds required to be cross-linked because it was readily soluble in water. Crosslinking led to reduction in hydrophilicity of polymer chains and preserved the scaffold integrity in aqueous media. Vapor cross-linking by glutaraldehyde was used to avoid the collapse of the PEO nanofibrous scaffolds during cross-linking in the aqueous system. Additionally, the unreacted aldehyde groups were treated with $0.1 \mathrm{~N}$ glycine aqueous solutions after vapor cross-linking. ${ }^{16}$

\section{Scanning electron microscopy (SEM)}

The SEM micrographs of plain PEO (2\%) (a), PEO $(3 \%)$ (b), PEO (4\%) (c), SIL loaded nanofibrous scaffolds SIL-NF2 (d), SIL-NF3 (e), SIL-NF4 (f) are shown in Figure 1. SEM micrographs showed that the plain PEO $(2 \%),(3 \%)$ and $(4 \%)$ solutions lead to formation of randomly oriented fibers. The electrospun scaffolds SIL-NF3 (image e) were comprised of randomly oriented fibers with a smooth and non-beaded morphology; this showed that the silybin is completely solubilized into the polymer matrix. Scaffolds SIL-NF2 and (image d) showed beaded morphology and SIL-NF4 (image f) showed slightly thick nanofibers. Polymer concentration is very important parameter in electrospinning. Morphology of formed nanofibers is dependent on viscosity of solution. At the low concentration of polymer, beaded structures are seen in the nanofibers and the process becomes electrospraying instead of electrospinning. ${ }^{20,21}$ Therefore, as the polymer concentration increased in SIL-NF3, no beads were observed in the as-spun nanofibers. In case of SIL-NF4, due to higher polymer concentration, solution viscosity increased slightly which might have led to hard ejection of nanofiber jet from solutions leading to thick fibers. ${ }^{22}$

Hence, from the above results, formulations SIL-NF3 was selected as optimized batch and further evaluated for DSC, XRD, Drug release kinetics, swelling and water 
uptake capacity, tensile strength and in-vivo wound healing studies.

\section{Differential scanning calorimetry (DSC) studies}

DSC thermograms of SIL, PEO, physical mixture of SIL and PEO and SIL-NF3 is depicted in Figure 2. DSC thermogram of SIL (curve a) exhibited a single endothermic peak at $164^{\circ} \mathrm{C}$, in agreement with the melting point and indicating its crystalline nature, whereas, DSC thermogram of PEO (curve b) was found in the range of $60-65^{\circ}$. Melting endotherm of SIL was not seen in thermogram of SIL-NF3 (curve d) indicating the drug was completely encapsulated inside the polymer matrix.

\section{X-ray diffraction studies (XRD)}

The XRD patterns of pure SIL, PEO and SIL-NF3 are shown in Figure 3. The diffraction pattern of SIL showed that it is highly crystalline in nature as indicated by its numerous distinctive peaks (Figure 3a). X-ray diffraction pattern of PEO also indicated its crystalline nature (Figure 3b). The characteristic peaks for SIL were absent in the diffraction pattern for SIL-NF3 indicating that SIL was in amorphous form in scaffold as presented in Figure 3d.

\section{Swelling behavior and water uptake capacity}

The capability to absorb exudates from wounds is important requirement for a dressing to be applied for healing wounds. Exudates in a wound promote the growth of bacteria Therefore, removal of exudates from the wound site is essential to protect the wound from mercerization. Excess exudate at wound site may lead to bacterial infection and consequently the wound becomes chronic. Hence, ability of scaffolds to absorb wound fluids is an important factor in wound healing. ${ }^{18}$ The degree of swelling and water uptake capacity (\%) for SIL-NF3 was found to be $11.70 \%$ and $6.39 \%$ respectively.

\section{Drug release kinetics studies}

The results of the in-vitro release behavior of SIL from SIL-NF3 scaffolds in physiological conditions are shown in Figure 4. The release profile (\%) showed initial burst release of drug (up to $12 \mathrm{~h}$ ). The SIL which was not entrapped in the polymer matrix remained on the surface and released quickly showing burst release. The second stage release can be attributed to the film degradation and diffusion of drug which might took place simultaneously. For the last stage of drug release, the release of drug molecules trapped inside the crystalline polymer structure may be responsible for the diffusion of drug from degrading scaffold structure. Drug release
(\%) from SIL-NF3 after $168 \mathrm{~h}$ was found to be $95.19 \%$. This results suggested that the prepared scaffolds could deliver the drug to the wound site efficiently over prolong period of time.

The release profile data were fitted into different mathematical models to analyze SIL release mechanism values of regression coefficient $\left(\mathrm{R}^{2}\right)$ were obtained from respective equations and given in Figure 4. Highest $R^{2}$ was obtained was found to be Korsmeyer-Peppas, with $\mathrm{R}^{2}=0.9912$ for SIL-NF3 scaffolds. Hence, KorsmeyerPeppas was considered as the best fitted model for SIL-NF3. From above studies, it was observed that the prepared scaffold showed diffusion and erosion based drug release profile.

\section{In vivo animal studies: Excision Wound Model Wound closure}

Wound closure can be a criterion for investigating wound healing visually. At similar time points, greater the wound closure better is the wound healing. The results for control, marketed formulation, SIL-NF3, NF-P, are shown in Figure 5. The results showed that the wound closure in marketed formulation, SIL-NF3 scaffold showed significant increase $(P<0.001)$ in \% wound closure at $3^{\text {rd }}, 7^{\text {th }}$ and $14^{\text {th }}$ day as compare to NS group. SIL-NF3 showed significant increase $(P<0.001)$ in $\%$ wound closure as compared to NF-P. Marketed formulation and SIL-NF3 showed comparable activity as depicted in Figure 5. Due to incorporation of Silybin in polymer structure of nanofibers, there is uniform deposition of SIL over wound area and enhanced the wound healing activity.

Figure 6 shows healing pattern upon application of NS $\left(a\right.$ and $\left.a_{1}\right)$ Marketed formulation treated $\left(b\right.$ and $\left.b_{1}\right)$, SIL-NF3 treated (c and $c_{1}$ ) plain NF treated $\left(\mathrm{d}_{\text {and }} \mathrm{d}_{1}\right)$ wounds at day 1 and day 14 .

In terms of overall external appearance, the wound closure appeared to be quite evident of the wound healing activity of particular formulation. The Figure 6 clearly showed that for SIL-NF3 and marketed formulation, the healing was very quick and the wounds were almost healed after $14^{\text {th }}$ day of scaffold application. Growth of epidermal layer from margin to center was faster which resulted in the contraction of wound area. Faster wound healing from SIL-NF3 can be attributed to the presence of SIL which have proven anti-oxidant and anti-inflammatory activity. SIL-NF3 showed better wound healing which may be due to the of nanofibrous structure which has larger surface area as well as porous structure and ability to mimic the fibrillar structure of extracellular matrix. ${ }^{23}$ Presence of nanofibrous structure in NF-P accelerated wound healing process compared 


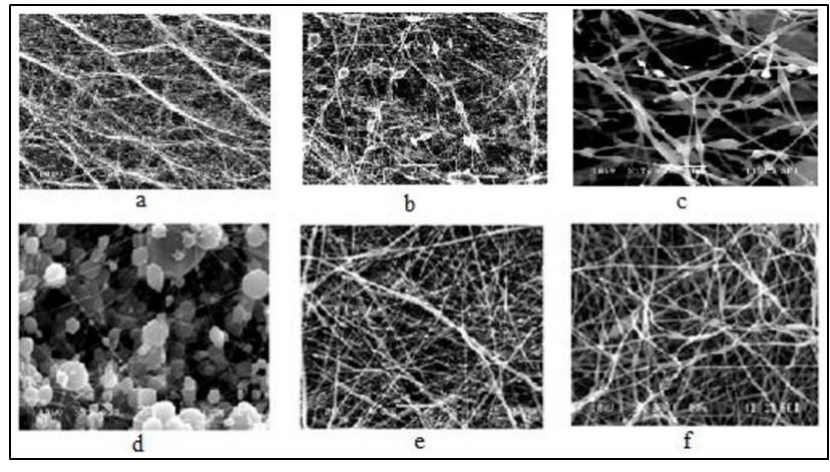

Figure 1: SEM micrographs of electrospun PEO (2\%) (a), PEO (3\%) (b), PEO (4\%) (c), SIL-NF2 (d), SIL-NF3 (e) and SIL-NF4 (f) scaffolds.

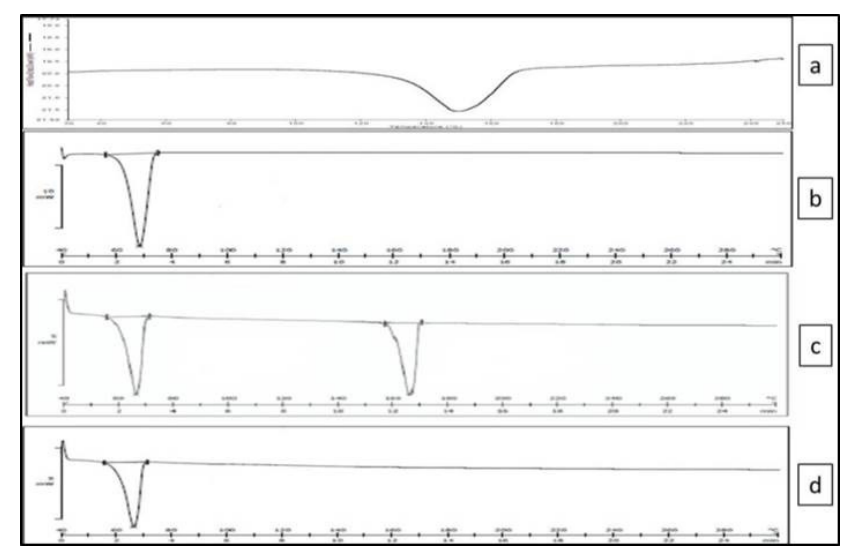

Figure 2: DSC thermograms of SIL (a), PEO (b), SIL and PEO physical mixture (c) and formulation SIL-NF3 (d).

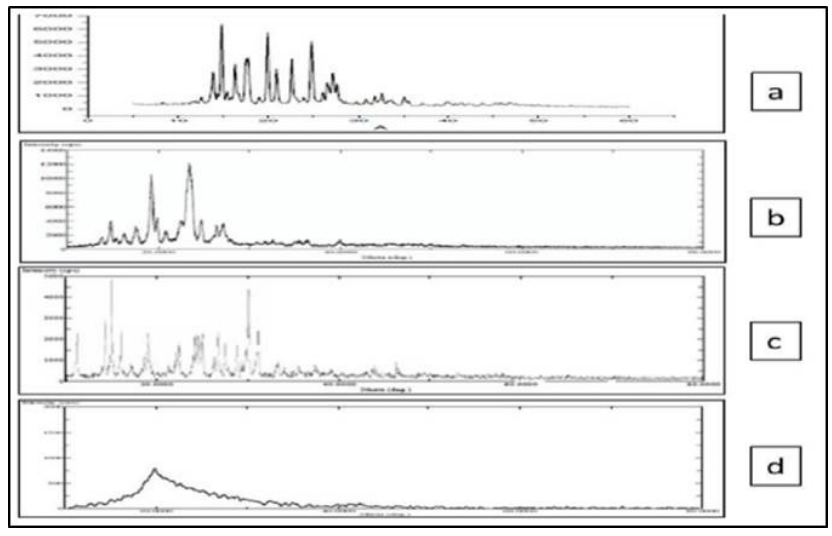

Figure 3: results for XRD studies of SIL (a), PEO (b), SIL and PEO physical mixture (c) and formulation SIL-NF3 (d).

with the control (group treated with NS) wounds. This may be attributed to the wound exudate absorbing properties of PEO.

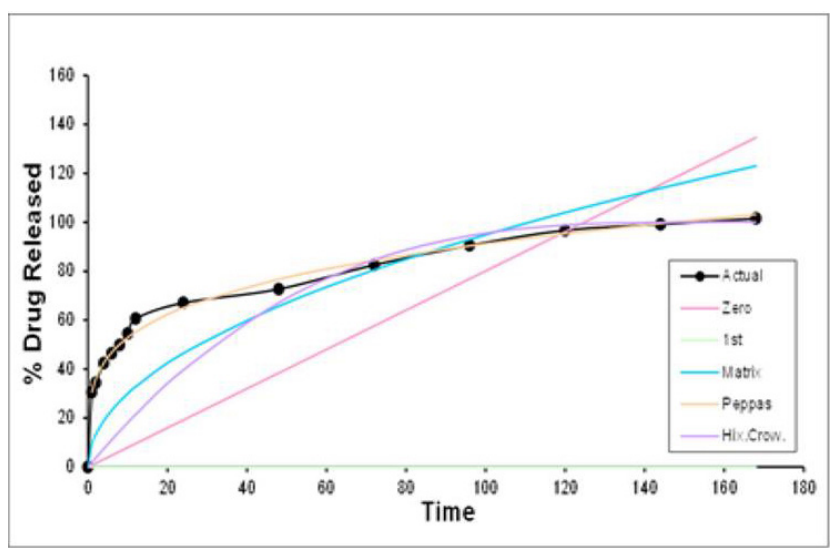

Figure 4: Drug release kinetic studies for SIL-NF3.

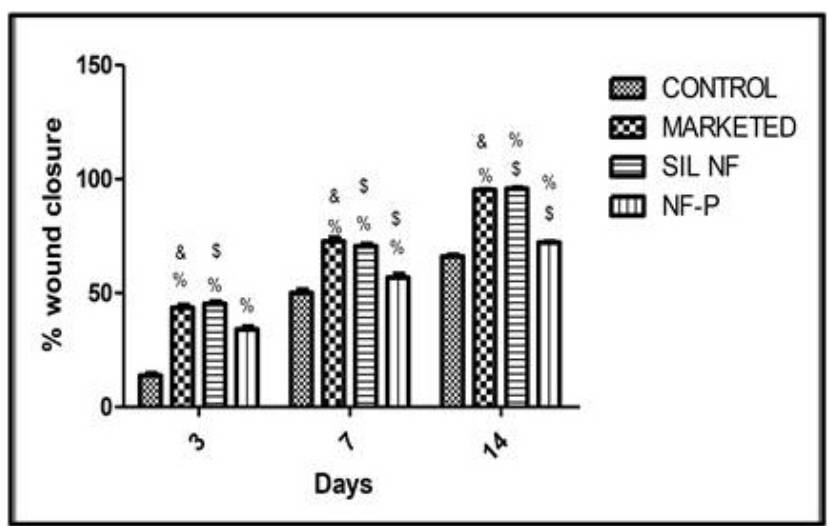

Figure 5: Results for \% wound closure studies.

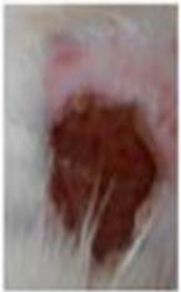

a

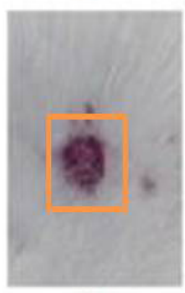

al

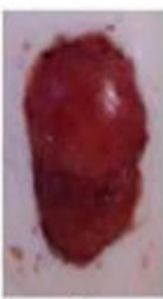

b

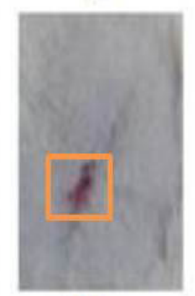

b1

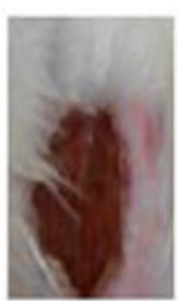

C

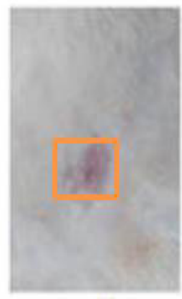

$\mathrm{cl}$

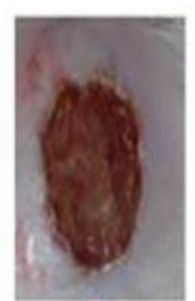

d

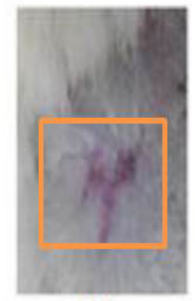

$\mathrm{d} 1$
Figure 6: Photographic images of wounds at $0^{\text {th }}$ day and $14^{\text {th }}$ day: control ( $a$ and $\left.a_{1}\right)$, marketed formulation $\left(b\right.$ and $\left.b_{1}\right)$, SIL-NF3 (c and $c_{1}$ ), plain-NF ( $d$ and $d_{1}$ ). 
a

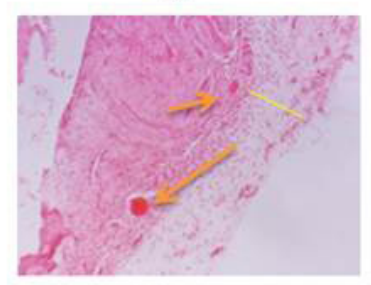

C

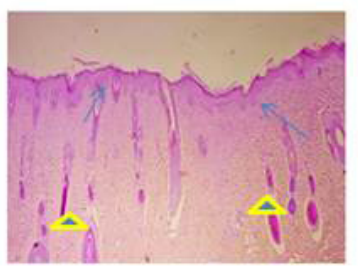

Figure 7: Hematoxylin and eosin histopathological images of wound tissue samples: control group (a), marketed formulation (b), SIL-NF3 (c), plain-NF (d) showing blood clots (orange arrow), fibrin layer (green line), newly formed epithelial layer (blue arrow), hair follicles (black arrow), epithelial layer in developing stage (green arrow) and regenerated blood vessels (yellow triangles).

\section{Histopathological evaluations}

The pathological images for HandE staining of wounds treated with NS (a), marketed formulation (b), SIL-NF3 (c), NF-P (d) are shown in Figure 7.

Figure $7 \mathrm{a}$ (control group treated with NS) indicates blood clots (yellow arrow) and fibrin layer (green line) which is in direct contact to the skin layer. In case of marketed formulation (Figure 7b), newly formed epithelial layer (blue arrow) is clearly visible. Figure 7c (SIL-NF3) shows well-developed epithelial layer (blue arrows) and abundance of newly formed small blood vessels in the dermis (yellow triangles). Section clearly shows growth of hair follicles (black arrow). Figure 7d (NF-P) depicts the partial formation of epithelial layer (blue arrows). Minute blood clots (orange arrow) and epithelial layer which is in still developing stage is also seen (green arrow).

\section{Reepithelization}

After 14 days, control specimen (group treated with NS) revealed only blood scabs and lot of inflammatory agents (Figure 7a). Fibrin layer was directly in contact with the wound surface and causes slower wound healing process. There was no evidence of epithelization in control (group treated with NS) specimen. Marketed formulation, SIL-NF3 treated groups (Figure 7b and 7c respectively) showed that both absorbed fibrin layers on the wounds and improved the healing process. Pathological studies showed that SIL loaded scaffolds improve the healing process while control group (treated with NS) specimen still need more time to heal.

In NF-P treated wounds, the epithelial layer was partially formed. As it is shown in histological image, there was remarkable epithelization $(\mathrm{P}<0.05)$ in the marketed, SIL-NF3, as well as NF-P treated wounds. While in the control (treated with NS) wound; there was complete absence of epithelial layer. It can be found that SIL-NF3 treated wounds disclosed better re-epithelization $(P<0.05)$ because of wound healing activity of silybin. In the plain NF-P scaffolds, epithelial layer was poorly formed and incomplete. In case of SIL-NF3 scaffolds, it can be predicted that SIL is beneficial to inhibit inflammatory ROS in wound tissues resulting in better wound healing. From these results we can conclude that the SIL-NF3 scaffold is beneficial in topical applications to detoxify inflammatory ROS and could prevent oxidative damage to skin tissue.

\section{Granular tissue score}

After histopathological studies, results were graded for granular tissue scores and resulted grades are presented in Table 2. Higher the granular tissue score better is the wound healing. The marketed formulation and treatment groups SIL-NF3, NF-P demonstrated higher granular tissue score as compared to control specimen (group treated with NS). From the data of granular tissue score, we can conclude that SIL-NF3 is efficient as marketed formulation.

\section{Wound maturity score}

In case of wound maturity score, results were graded and resulted grades are presented in Table 2. Marketed formulation, SIL-NF3, NF-P treated groups showed higher wound maturity scores when compared to control group (treated with NS) wounds. Marketed formulation and SIL-NF3 treated wounds show comparable response in terms of wound maturity score.

\section{Collagen distribution}

\begin{tabular}{|c|c|c|c|}
\hline \multicolumn{3}{|c|}{ Table 2: Results for histological evaluations. } \\
\hline Group & $\begin{array}{c}\text { Period of } \\
\text { epithelisation } \\
\text { (Days) }\end{array}$ & $\begin{array}{c}\text { Granular } \\
\text { tissue score }\end{array}$ & $\begin{array}{c}\text { Wound } \\
\text { maturity } \\
\text { score }\end{array}$ \\
\hline $\begin{array}{c}\text { Control } \\
\text { Marketed }\end{array}$ & $33.15 \pm 0.10$ & $1.1 \pm 0.23$ & $1.2 \pm 0.91$ \\
\hline SIL-NF3 & $19.22 \pm 0.85^{\%}$ & $4.86 \pm 0.32^{\%}$ & $4.84 \pm 0.5 \%$ \\
\hline Plain-NF & $25.50 \pm 0.54^{* a}$ & $3.04 \pm 0.12^{\% a}$ & $3.34 \pm 0.63^{\% a}$ \\
\hline
\end{tabular}

$\mathrm{n}=6$; values are expressed as mean \pm SEM: One-way ANOVA followed by Tukey's test. ${ }^{\%} P<0.05$ vs control, ${ }^{*} P<0.05$ Vs NF-P, a $P<0.05$ Vs marketed. 
a

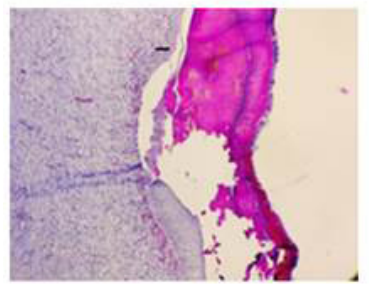

C

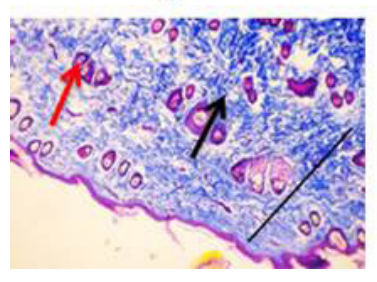

Figure 8: Masson's trichrome staining histopathological images of wound tissue samples: control group (a), marketed formulation (b), SIL-NF3 (c) and plain-NF (d) showing newly formed blood vessels (red arrow) and regenerated collagen (black arrow).

As it is shown in Figure 8, after 15 days, the control group treated with NS (image a) showed very minute collagen regeneration. Marketed formulation (B), SIL-NF3 (C) showed better collagenous regeneration as compared to NF-P (D) scaffolds. Figure 8a control group (treated with NS) indicates non-uniform epithelial layer. In case of marketed formulation (Figure 8b) newly formed collagen fibers (black arrow) and formed small blood vessels (red arrows) are clearly seen. In Figure 8c (SIL-NF3), intensity of blue colour determines the abundance of newly formed collagen (black arrow). Small blood vessels (red arrow) are clearly visible. Figure 8d (NF-P) shows minute collagenous regeneration.

The wounds that were treated with SIL-NF3 showed newly formed collagen in bundle shape with an irregular pattern which signifies the efficiency of silybin scaffolds in wound healing. Granulation tissues with some skin appendages such as hair follicles are seen in the SILNF3 and marketed formulation treated wounds. The SIL-NF3 scaffold when compared with marketed formulation, it was found that, the SIL-NF3 scaffold showed relatively similar uniformity and distribution of collagen as that of marketed formulation. These considerable improvements could be due to wound healing activity of SIL.

\section{CONCLUSION}

SIL loaded scaffolds were successfully prepared by electrospinning process. Scaffold fabrication technique is simple and reproducible without use of any com- plicated instruments. Characterization studies revealed formation of water-stable structure. Swelling and water uptake behaviour of scaffolds is optimum for wound exudate absorption and for exchange of gases. The SIL release from the scaffolds was found to be slow and sustained with biphasic behaviour for prolong period of time which avoids frequent application of scaffolds to the wound site. Finally, the in vivo wound healing study revealed that the SIL loaded scaffolds show faster wound healing as compared to control (treated with NS) and plain scaffolds. The study confirms the ability of SIL to promote wound healing qualitatively and quantitatively. It can be concluded that SIL enriched PEO nanofibrous scaffold is beneficial in topical applications to form the basis for new skin regenerating and wound healing.

\section{ACKNOWLEDGMENT}

Authors are thankful to Mr Satishchandra Ogale and Mr Rounak Nafade (CSIR-NCL, Pune) for their valuable guidance regarding electrospinning.

\section{CONFLICT OF INTEREST}

The authors declare no conflict of interest.

\section{ABBREVIATIONS}

SIL: Silybin; PEO: Polyethylene oxide, SEM: Scanning electron microscopy, DSC: Differential scanning calorimetry, XRD: X-ray diffraction, DCM: Dichloromethane, DMF: Dimethyl formamide, ROS: Reactive oxygen species, SOD: Superoxide dismutase, GPx: Glutathione peroxidase, COX-2: Cyclooxygenase-2, IAEC: Institutional Animal Ethics Committee, CPCSEA: Committee for Purpose of Control and Supervision of Experiments on Animals, HPLC: High performance liquid chromatography, NF2-P: Plain nanofibrous scaffold with $2 \%$ PEO, NF3-P: Plain nanofibrous scaffold with 3\% PEO, NF4-P: Plain nanofibrous scaffold with 4\% PEO, SIL-NF2: Silybin loaded nanofibrous scaffold with 2\% PEO, SIL-NF3: Silybin loaded nanofibrous scaffold with 3\% PEO, SIL-NF4: Silybin loaded nanofibrous scaffold with 4\% PEO, $\mathbf{H}$ and E: Hematoxyline and eosin, MTS: Masson's trichrome staining, ANOVA: Analysis of variance.

\section{REFERENCES}

1. Monteiro N, Martins M, Martins A, Fonseca N, Moreire J, Reis R, et al. Antibacterial activity of chitosan nanofiber meshes with liposomes immobilized releasing gentamicin. Acta Biomaterialia. 2015;18:196-205. 
2. Radhakumary C, Antony M, Sreenivasan K. Drug loaded thermoresponsive and cytocompatible chitosan based hydrogel as potential wound dressing. Carbohydrate Polymers. 2011;83(2):705-13.

3. Elsner J, Zilberman M. Antibiotic eluting bioresorbable composite fibers for wound healing applications: Microstructure, drug delivery and mechanical properties. Acta Biomateriala. 2009;5(8):2872-83.

4. Singh B, Sharma S, Dhiman A. Design of antibiotic containing hydrogel wound dressings: Biomedical properties and histological study of wound healing. International Journal of Pharmaceutics. 2013;457(1):82-91.

5. Pawar H, Tetteh J, Boateng J. Preparation, optimization and characterization of novel wound healing film dressing loaded with streptomycin and Diclofenac. Colloid and Surfaces B: Biointerfaces. 2013;102:102-10.

6. Ruzickova J, Velebny V, Novak J, Szuszkiewicz K, Knotkova K, Foglarova M, et al. Hyaluronic acid based nanofibers for wound dressing and drug delivery carriers. Fundamental Biomedical Technologies. 2014;7:417-33.

7. Morie A, Garg T, Goyal A, Rath G. Nanofibers as novel drug delivery carriersAn overview. Artificial Cells, Nanomedicine and Biotechnology. 2014;1-9.

8. Jia Y, Gong J, Gu X, Kim H, Dong J, Shen X. Fabrication and characterization of poly(vinyl alcohol)/chitosan blend nanofibers produced by electrospinning method. Carbohydrate Polymers. 2007;67(3):403-9.

9. Hu X, Lui S, Zhou G, Huang Y, Xie Z, Jing X. Electrospinning of polymeric nanofibers for drug delivery applications. Journal of Control Release. 2014;185:12-21.

10. Schiffman J, Schauer C. A review: Electrospinning of biopolymer nanofibers and their applications. Polymer Reviews. 2008;48(2):317-52.

11. Svobodova A, Walterova D, Psotova J. Influence of silymarin and its flavonolignans on $\mathrm{H}_{2} \mathrm{O}_{2}$ induced oxidative stress in human keratinocytes and mouse fibroblasts. Burns. 2006;32(8):973-9.

12. Tabandeh M, Oryan A, Alipour A, Naieni A. Silibinin regulates matrix metalloproteinase 3 (stromelysine1) gene expression, hexoseamines and collagen production during rat skin wound healing. Phytotherapy Research. 2013;27(8):1149-53.
13. Singh R, Agarwal R. Cosmeceuticals and silibinin. Clin Dermatol. 2009;27(5):479-84.

14. Shrotriya SN, Vidhate BV, Shukla MS. Formulation and development of Silybin loaded solid lipid nanoparticle enriched gel for irritant contact dermatitis. Journal of Drug Delivery Science and Technology. 2017.

15. Har-el Y, Gerstenhaber J, Brodsky R, Huneke R, Lelkes P. Electrospun soy protein scaffolds as wound dressings: Enhanced reepithelialization in a porcine model of wound healing. Wound Medicine. 2014;5:9-15.

16. Rho K, Jeong L, Lee G, Seo B, Park Y, Hong S, et al. Electrospinning of collagen nanofibers: Effects on the behaviour of normal human keratinocytes and early-stage wound healing. Biomaterials. 2006;27(8):1452-61.

17. Kasoju N, Bora U. Fabrication and characterization of curcumin releasing silk fibroin scaffold. J Biomed Mater Res Part B. 2012;B:1-13.

18. Alam A, Quazi S. Surface modified thin film from silk and gelatin for sustained drug release to heal wound. Journal of Material Chemistry B. 2015;3(31):6473-9.

19. Siritienthong T, Ratanavaraporn J, Aramwit P. Development ethyl alcohol precipitated silk sericin/polyvinyl alcohol scaffolds for accelerated healing of full-thickness wounds. International Journal of Pharmaceutics. 2012;439(1-2): 175-86.

20. Kanani A, Bahrami S, Joghataie M, Samadikuchaksaraei A, Ahmadi-Taftie H, Rabbani S, et al. Tissue engineered poly(caprolactone)-chitosan-poly(vinyl alcohol) nanofibrous scaffolds for burn and cutting wound healing. IET Nanobiotechnology 2014;8(2):123-31.

21. Lui $\mathrm{Y}, \mathrm{He} \mathrm{J}, \mathrm{Yu} \mathrm{J}$, Zeng $\mathrm{H}$. Controlling numbers and sizes of beads in electrospun nanofibers. Polymer International. 2008;57(4):632-6.

22. Li Z, Wang C. Effects of working parameters on electrospinning. One-Directional Nanostructures. 2013;15-28.

23. Tamm I, Heinamaki J, laidmae I, Rammo L, Paaver U, Ingebrigsten S, et al. Development of suberin fatty acids and chloramphenicol-loaded antimicrobial electrospun nanofibrous mats intended for wound therapy. Journal of Pharmaceutical Sciences. 2016;105(3):1.

\section{PICTORIAL ABSTRACT}

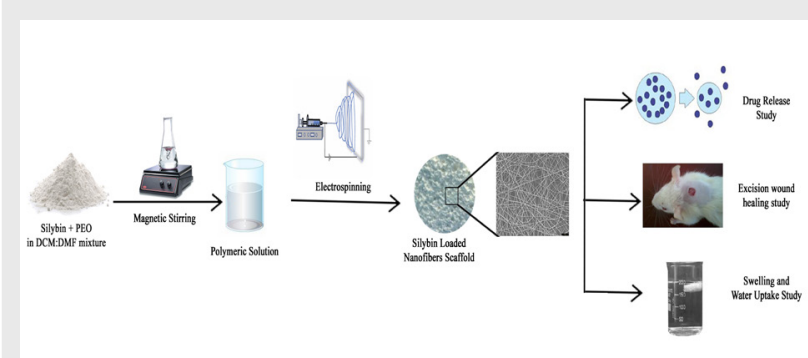

\section{SUMMARY}

- Poly (ethylene oxide) was selected (3\%) in DCM: DMF (8:2) mixture and nanofibers were prepared by electrospinning. The scaffolds were further crosslinked with glutaraldehyde to increase tensile strength of scaffolds.

- TheoptimizedSIL-NF3scaffoldshoweddrugrelease of $95.19 \%$ in $168 \mathrm{~h}$ respectively indicating controlled release of silybin through scaffolds. This results suggested that the prepared scaffolds could deliver the drug to the wound site efficiently over prolong period of time.

- Wound healing studies suggested that the prepared scaffolds facilitate healing of wounds at early stage. Therefore, the polymeric electrospun scaffold are proven as highly effective carriers for wound exudate absorption, drug deposition which is desirable for wound healing.

Cite this article: Kapadnis PD, Shrotriya SN. Electrospun Silybin Enriched Scaffolds of Polyethylene Oxide as Wound Dressings: Enhanced Wound Closure, Reepithelization in Rat Excisional Wound Model. Indian J of Pharmaceutical Education and Research. 2019;53(2):301-9. 